\title{
Model for investigating snorers with suspected sleep apnoea
}

\author{
Helmuth Rauscher, Wolfgang Popp, Hartmut Zwick
}

\begin{abstract}
Background Overnight polysomnography is expensive and time consuming. An approach based on a logistic regression model and overnight pulse oximetry has been developed to determine which of the snorers referred to our sleep laboratory need polysomnography.

Methods The variables entered in the regression model were derived from questionnaires completed by 95 habitual snorers and 89 patients with obstructive sleep apnoea. The resulting regression equation included weight, height, sex, witnessed episodes of apnoea, and reports of falling asleep when reading. This prediction equation was applied to a sample of 116 consecutive patients referred for investigation of heavy snoring. Pulse oximetry data on the 116 test subjets were obtained during polysomnography and analysed separately. Pulse oximetry was judged to indicate obstructive sleep apnoea when it showed cyclic oscillations of oxyhaemoglobin saturation or heart rate, or both, for more than 30 minutes during the study night.
\end{abstract}

Results A cut off probability of 0.31 gave the prediction model a sensitivity of 94\% to predict an apnoea-hypopnoea index above 10 , with a specificity of $45 \%$. When this cutoff point was used to predict an apnoea-hypopnoea index of over 20 sensitivity was $95 \%$ and specificity $41 \%$. Combined with oximetry our regression model had a sensitivity of $100 \%$ for predicting an apnoea-hypopnoea index of more than 10 . On the other hand, all patients with negative results from oximetry and a probability value below 0.31 had an apnoea-hypopnoea index lower than 10 according to polysomnography.

Conclusions It is concluded that snorers with negative results from oximetry classified as not having obstructive sleep apnoea according to this model do not need polysomnography.

(Thorax 1993;48:275-279)

Obstructive sleep apnoea is a common problem among middle aged obese snorers. ${ }^{12}$ Although complaints about loud snoring, daytime sleepiness, and psychomental impairment are characteristic features of obstructive sleep apnoea, these symptoms also occur in habitual snorers without sleep apnoea. ${ }^{34}$ As the development from snoring to obstructive sleep apnoea is gradual, even apnoea reported by the bed partner does not clearly differentiate between snorers with and without clinically relevant obstructive sleep apnoea. Increasing awareness of the risks associated with obstructive sleep apnoea in regard to morbidity and mortality ${ }^{5-8}$ has led to a rapid rise in the number of referrals to sleep laboratories. Thus several attempts have been made to identify those patients in need of a sleep study among the large number of snorers seeking medical help for their problem. Whereas a scoring system based on self reporting ${ }^{9}$ detected obstructive sleep apnoea in around $80 \%$ of cases, a recently published regression model ${ }^{10}$ based on age, weight, height, blood pressure, and reported episodes of apnoea predicted more than 15 disturbed breathing events per hour of sleep with a sensitivity of $92 \%$ and a specificity of $51 \%$.

Because of the low specificity, however, such models are of limited value in reducing the number of polysomnographic studies in snorers without obstructive sleep apnoea. Furthermore, relying completely on such a prediction model carries the risk of missing some patients with obstructive sleep apnoea. To compensate for the shortcomings of a prediction model we have combined it with pulse oximetry. As most episodes of apnoea are associated with cyclic oscillations of oxyhaemoglobin saturation $\left(\mathrm{SaO}_{2}\right)$ and pulse rate ${ }^{11} 12$ typical obstructive sleep apnoea is easily recognisable from overnight pulse oximetry. ${ }^{1314}$ The sensitivity of oximetry may, however, be poor in patients with obstructive sleep apnoea who have short episodes of apnoea and only minor desaturations ${ }^{13-16}$ and in snorers with incomplete upper airway obstruction that is not associated with apnoea or desaturation but may lead to severe fragmentation of sleep. ${ }^{3}$ As both conditions usually cause daytime sleepiness these patients should be detectable from a prediction model that includes some feature of excessive daytime sleepiness. We report a series of snorers referred to a sleep laboratory because of suspected obstructive sleep apnoea on which these ideas have been tested.

\section{Methods}

To develop the regression model we used data from 95 habitual snorers with an apnoea-hypopnoea index below 10 and from 89 patients with obstructive sleep apnoea

\footnotetext{
Returned to authors
May 1992

Revised version received

26 May 1992

Accepted 9 July 1992

Department, Austria

W Popp

H Zwick

Reprint requests to: Dr H Rauscher
} 
Table 1 Anthropometric data (mean (SD) values) on 89 patients with obstructive sleep apnoea (OSA) and 95 habitual snorers used to develop the regression model

\begin{tabular}{lll}
\hline & Habitual snorers & OSA \\
\hline Age (y) & $49 \cdot 0(11 \cdot 7)$ & $50 \cdot 6(10 \cdot 2)$ \\
Body mass index & $28 \cdot 4(3 \cdot 1)$ & $31 \cdot 8(5 \cdot 3)^{\star}$ \\
Male : female & $63: 32$ & $75: 14$ \\
\hline
\end{tabular}

${ }^{\star} \mathrm{p}<0.001$

with an apnoea-hypopnoea index above 20 . These 184 subjects were part of a population of 222 snorers referred to our sleep laboratory for investigation of suspected obstructive sleep apnoea during the course of one year. The 38 subjects among this population with an apnoea-hypopnoea index of 10-20 were excluded so that we would have two clearly distinct groups-one with obstructive sleep apnoea and one with heavy snoring only. Anthropometric data on the study population are given in table 1 . Most of our patients $(63 \%)$ were self referred because they had read about snoring and sleep apnoea; the others were referred by their ear, nose, and throat specialist $(17 \%)$, their general practitioner $(14 \%)$, or other specialist $(6 \%)$.

All patients underwent all night polysomnography, including electroencephalography, electro-oculography, submental electromyography, electrocardiography, measurement of airflow at the nose and mouth and of the movements of rib cage and abdomen, and oximetry (Minolta Pulsox 7). These variables were recorded by a computer using the Respisomnographe hardware and software (SEFAM, Vandoeuvre-Les-Nancy, France) and were manually scored according to standard criteria. ${ }^{17}$ An episode of apnoea was defined as cessation of airflow at the nose and mouth for longer than 10 seconds. Hypopnoea was defined as a reduction in rib cage and abdominal movements to $50 \%$ or less of those recorded during the preceding five breaths for longer than 10 seconds, accompanied by a fall in $\mathrm{SaO}_{2}$ to $92 \%$ or lower if baseline was $94 \%$ or more or by a fall in $\mathrm{SaO}_{2}$ of $3 \%$ or more if baseline was $93 \%$ or less. The total number of episodes of apnoea and hypopnoea per hour of sleep represented the apnoea-hypopnoea index.

The symptoms and complaints of all patients were recorded by a self administered questionnaire with 36 items, most of them requiring simple "yes or no" decisions. The frequencies of positive and negative answers given by the two groups were compared by $\chi^{2}$ test and a significant difference was assumed at $\mathrm{p}$ values below $0 \cdot 05$. Ordered according to decreasing $\chi^{2}$ value, all items with significant differences were entered in a stepwise logistic regression analysis using the BMDP statistical software package for microcomputers. ${ }^{18}$ The variables entering the model did not change when we also included the data on the 38 patients with an apnoea-hypopnoea index of 10-20.

The resulting regression equation was then applied to another sample-116 consecutive patients (95 men, 21 women) referred for investigation of heavy snoring. According to polysomnography 47 of them had an apnoeahypopnoea index above 10 and 38 above 20 . The pulse oximetry data for these subjects were analysed separated from polysomnography by an investigator blinded to the patient's symptoms and the polysomnography results. For scoring of pulse oximetry the full outprints of our Sleepox software ${ }^{19}$ were used, where intervals of 30 minutes are displayed on a $13 \mathrm{~cm}$ width of print (theoretically this would represent a paper speed of $6.5 \mathrm{~cm} / \mathrm{h}$ on a conventional chart recorder). The pulse oximetry data were considered positive for obstructive sleep apnoea if the sum of all intervals during the study night showing cyclic oscillations of $\mathrm{SaO}_{2}$ or pulse rate exceeded 30 minutes. Scoring was based solely on pattern recognition without definition of limits for the amplitudes of oscillations in $\mathrm{SaO}_{2}$ and pulse rate.

\section{Results}

The symptoms and complaints of the two patient groups used to develop the model are

Table 2 Symptoms and complaints of the 89 patients with obstructive sleep apnoea (OSA) and 95 habitual snorers (HS) used to develop the regression model and the 47 subjects with an apnoea-hypopnoea index (AHI) above 10 and 69 subjects with an AHI lower than 10 used to test the model

\begin{tabular}{|c|c|c|c|c|c|c|}
\hline & \multirow{2}{*}{$\begin{array}{l}\text { OSA } \\
(\%)\end{array}$} & \multirow{2}{*}{$\begin{array}{l}H S \\
(\%)\end{array}$} & \multirow[b]{2}{*}{$\chi^{2}$} & \multicolumn{2}{|l|}{$A H I$} & \multirow[b]{2}{*}{$\chi^{2}$} \\
\hline & & & & $\geqslant 10(\%)$ & $<10(\%)$ & \\
\hline Reported episodes of apnoea & 73 & 44 & 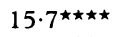 & 77 & 46 & $10 \cdot 5^{\star \star \star}$ \\
\hline$>20 \%$ overweight & 84 & 66 & 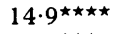 & 62 & 42 & $4 \cdot 3^{\star}$ \\
\hline Hypertension & 62 & 39 & $9 \cdot 6^{\star \star \star}$ & 55 & 36 & $4 \cdot 1^{\star}$ \\
\hline Falling asleep when driving & 28 & 11 & $9 \cdot 2^{\star \star \star}$ & 26 & 7 & $7 \cdot 5^{\star}$ \\
\hline Falling asleep after nocturnal awakenings within $10 \mathrm{~min}$ & 66 & 45 & $8 \cdot 2^{\star \star \star}$ & 68 & 44 & $6 \cdot 8^{\star}$ \\
\hline Nocturia & 64 & 43 & $8 \cdot 1^{\star \star \star}$ & 79 & 56 & $6 \cdot 0^{\star}$ \\
\hline Male gender & 84 & 66 & $7 \cdot 9^{\star \star \star}$ & 87 & 78 & 1.5 \\
\hline Falling asleep when reading & 60 & 39 & $7 \cdot 8^{\star \star \star}$ & 57 & 36 & $5 \cdot 1^{\star}$ \\
\hline Falling asleep when talking & 17 & 6 & $5 \cdot 1^{\star}$ & 13 & 9 & $0 \cdot 5$ \\
\hline Falling asleep when watching TV & 82 & 68 & $4 \cdot 5^{\star}$ & 81 & 67 & $2 \cdot 8$ \\
\hline Restless sleep & 48 & 34 & $4 \cdot 1^{\star}$ & 43 & 35 & $0 \cdot 7$ \\
\hline Waking up choking & 33 & 20 & $3 \cdot 8$ & 15 & 28 & $2 \cdot 6$ \\
\hline Falling asleep at bedtime within $10 \mathrm{~min}$ & 61 & 47 & $3 \cdot 3$ & 75 & 41 & $12 \cdot 9^{\star \star \star}$ \\
\hline Poor mental performance & 58 & 45 & $3 \cdot 2$ & 43 & 49 & 0.5 \\
\hline Feeling sleepy during daytime & 64 & 55 & $1 \cdot 7$ & 70 & 55 & $2 \cdot 7$ \\
\hline Hearing own snoring on awakening & 33 & 25 & $1 \cdot 2$ & 23 & 28 & $0 \cdot 3$ \\
\hline
\end{tabular}

${ }^{\star} \mathrm{p}<0.05 ;{ }^{\star \star} \mathrm{p}<0.01 ;{ }^{\star \star \star} \mathrm{p}<0.005 ;{ }^{\star \star \star \star} \mathrm{p}<0.001$ 
Table 3 Distribution of the 116 test subjects in groups with probability values ( $p$ ) for obstructive sleep apnoea (OSA) above and below the cutoff point of 0.31 according to apnoea-hypopnoea index (AHI) and gender

\begin{tabular}{lrrrrrrr}
\hline & \multicolumn{2}{l}{$p<0 \cdot 31$} & & \multicolumn{2}{c}{$p \geqslant 0 \cdot 31$} \\
\cline { 2 - 4 } \cline { 6 - 8 } AHI & $M$ & $F$ & Total & & $M$ & $F$ & Total \\
\hline$<10$ & 20 & 11 & 31 & & 34 & 4 & 38 \\
$\geqslant 10$ & 1 & 2 & 3 & & 40 & 4 & 44 \\
$<20$ & 21 & 11 & 32 & & 42 & 4 & 46 \\
$\geqslant 20$ & 0 & 2 & 2 & & 32 & 4 & 36 \\
\hline
\end{tabular}

given in table 2. Of the 11 items found to have different frequencies in the two groups, only four contributed significantly to the logistic regression model. Thus the equation found to estimate probability of obstructive sleep apnoea in heavy snorers was:

$$
\text { Probability }=\frac{\mathrm{e}^{\mathrm{k}}}{1+\mathrm{e}^{\mathrm{k}}},
$$

where $\mathrm{k}=(-7 \cdot 263-2 \cdot 046 a+0.0487 b+$ $1 \cdot 121 c+1 \cdot 663 d)$ and $a=0$ if male and 1 if female, $b=$ weight in $\mathrm{kg} \times 100 /$ height in $\mathrm{cm}$ $-100, c=1$ if falling asleep when reading is reported and 0 if not, $d=1$ if episodes of apnoea are reported and 0 if not.

Although a cut off probability of 0.56 had the highest efficacy (= true positive predictions plus true negative predictions divided by total number of study subjects), with $77 \%$ of the patients classified correctly, the sensitivity in predicting an apnoea-hypopnoea index of 10 or more was too poor for clinical use. Thus we chose a cut off point of 0.31 as the

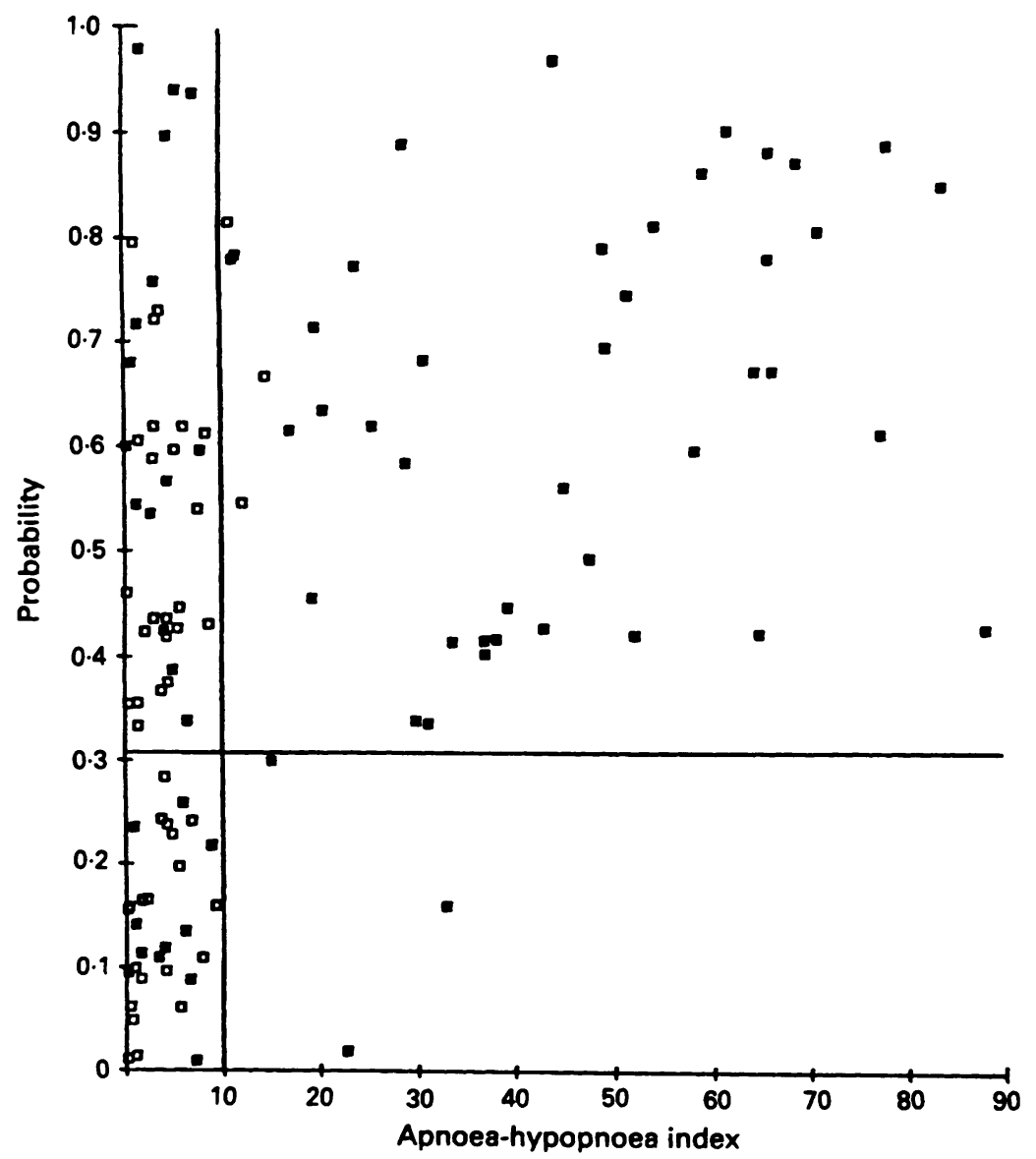

Probability values calculated from the regression model for 116 snorers as a function of the apnoea-hypopnoea index. Full squares indicate subjects with overnight oximetry giving results positive for obstructive sleep apnoea and open squares those with negative results. best compromise between high sensitivity and relatively well preserved specificity.

The sensitivity of our model for the detection of obstructive sleep apnoea was defined as the number of true positive predictions divided by the number of patients with an apnoea-hypopnoea index of 10 or more or 20 or more. Specificity was defined as the number of true negative predictions divided by the number of patients with an apnoea-hypopnoea index below 10 or 20 . With our equation applied to the 116 test subjects (table 3), this cut off point gave the model a sensitivity of $94 \%$ for predicting an index of 10 or more (three false negatives out of 47 ) with a specificity of $45 \%$ (31 true negatives out of 69 ). When we used this cut off point to predict an apnoea-hypopnoea index of 20 or more sensitivity was $95 \%$ and specificity $41 \%$. The negative predictive value (=true negative predictions divided by total negative predictions) of a probability below 0.31 was $91 \%$ for an apnoea-hypopnoea index of 10 or less and $94 \%$ for one of 20 or less. As can be seen from table 3, the model performed better for men than for women, the sensitivity for predicting an apnoea-hypopnoea index of 20 or more in men being $100 \%$. The symptoms and complaints of these 116 test subjects are also given in table 2 .

Three patients with more than 10 episodes of apnoea per hour of sleep were missed by our model. The first was a 30 year old woman with a body mass index of 20.9 , who reported heavy snoring and excessive daytime sleepiness but had no witnessed episodes of apnoea. The apnoea-hypopnoea index from polysomnography was 22.7 and ear, nose, and throat examination showed enlarged tonsils and an abnormally long soft palate with a large uvula. The polysomnogram obtained two months after surgery showed complete disappearance of sleep disordered breathing. The second patient was a 77 year old woman of normal weight with an apnoea-hypopnoea index of 32.9. Although she reported episodes of apnoea and sleepiness the probability calculated from our model was only $0 \cdot 16$. The third case missed by our model was a 26 year old man with a body mass index of $24 \cdot 1$, who complained about daytime sleepiness but had no witnessed episodes of apnoea. Whereas his probability value was $0 \cdot 3$, polysomnography yielded an apnoeahypopnoea index of 15 .

Pathological results from oximetry, according to our definition, were found in 70 patients, in 26 of whom simultaneous polysomnography showed an apnoea-hypopnoea index lower than 10 . Only three patients with an apnoea-hypopnoea index of 10 or more had negative results from oximetry. Thus sensitivity for detecting obstructive sleep apnoea from more than 30 minutes of cyclic oscillations in $\mathrm{SaO}_{2}$ or pulse rate during the night was $94 \%$ and specificity $62 \%$.

As shown in the figure, the three patients considered as not having vostructive sleep apnoea on the basis of oximetry were in the high probability group, and the three patients 
from the low probability group with an apnoea-hypopnoea index of 10 or more had positive results from oximetry. Thus the combination of oximetry with our regression model had a sensitivity of $100 \%$ for predicting an apnoea-hypopnoea index of 10 or more. On the other hand, all patients with negative results from oximetry in the low probability group had an apnoea-hypopnoea index below 10. Thus, despite the low overall specificity for obstructive sleep apnoea, our approach makes possible a reduction of negative polysomnograms of $29 \%$ (49 instead of 69) without the risk of missing patients with obstructive sleep apnoea.

\section{Discussion}

The purpose of this study was to develop an approach to the investigation of snorers referred to a sleep laboratory with possible obstructive sleep apnoea. Our goal was to reduce the number of polysomnography studies with a negative result in those who only snore-without missing patients with clinically relevant sleep disordered breathing. As we think it inappropriate to refuse a sleep study-for example, in a snorer with witnessed episodes of apnoea-solely because of the lack of relevant clinical symptoms, we chose pulse oximetry as a simple and widely available screening test for obstructive sleep apnoea as our first step. The decision about further investigation could then be based on the results of oximetry and the probability of obstructive sleep apnoea calculated from a logistic regression model.

The variables entering our prediction model were weight, height, sex, witnessed apnoea, and the positive or negative answer to the question: "Do you frequently fall asleep during the day when reading books or newspapers?" Using our regression equation with a cut off probability value of 0.31 , we were able to predict an apnoea-hypopnoea index of 10 or more with a sensitivity of $94 \%$ in 116 unselected heavy snorers. Only three of the 47 subjects with an apnoea-hypopnoea index of 10 or more were misclassified by our regression equation. These patients were rather different from the typical patient with obstructive sleep apnoea, for whom our prediction model worked very well, and all of them had pathological results from pulse oximetry according to our definition.

In contrast to a recently published approach for predicting sleep apnoea from clinical symptoms, ${ }^{10}$ our model included a variable indicating excessive daytime sleepiness but did not include age or hypertension. Although hypertension was more frequently found in patients with obstructive sleep apnoea than in habitual snorers, its prevalence was not correlated with severity of sleep disordered breathing and was influenced only by obesity and male gender-both included in our model. The reason for not including age was that we found neither an increase in apnoea-hypopnoea index with age $(r=0.02)$ nor a higher prevalence of obstructive sleep apnoea in older age groups, which may be due to the high percentage of self referrals among our study population, resulting in an overrepresentation of young patients with severe obstructive sleep apnoea by comparison with the general population. Surprisingly, questions about typical features of obstructive sleep apnoea, such as short sleep latency, restless sleep, awakenings with choking, or difficulty in staying awake in situations other than reading, did not enter the model by stepwise logistic regression analysis. As our model is thought to estimate the probability of obstructive sleep apnoea in snorers and not to discover patients with obstructive sleep apnoea among the general population, snoring is not included in the equation. Snoring, however, is the most common finding in patients with obstructive sleep apnoea, so a patient's complaint of loud snoring means that obstructive sleep apnoea should be excluded. Our approach should be helpful in predicting 10 or more sleep disordered breathing events per hour of sleep, which is well below the apnoea index associated with increased mortality. ${ }^{5}$ How generally applicable our model is remains to be established. The method of collecting data-that is, type of questionnaire or questionnaire versus interview-may influence the frequency with which subjective complaints are elicited. For clinical purposes our equation can be recommended only when used with pulse oximetry, whose shortcomings ${ }^{1314}$ it overcomes.

From our results we conclude that patients in the low probability group classified as not having obstructive sleep apnoea on the basis of oximetry do not need polysomnography. Firstly, they generally have only very mild or no symptoms and, secondly, our definition of pathological oximetry results was rather rigorous. A total of 30 minutes of cyclic oscillations of $\mathrm{SaO}_{2}$ or pulse rate means that, for example, for a sleep time of six hours there would be an apnoea-hypopnoea index of about 5-8. Thus even patients with supine or sleep stage dependent obstructive sleep apnoea are unlikely to be missed. On the other hand, this definition resulted in a relatively high number of false positive results from oximetry, which contrasts with studies using less rigorous definitions of abnormality. ${ }^{13} 14$

Our plan of investigation should be capable of reducing the number of polysomnography studies with a negative result in snorers by about one third (49 instead of 69 in our 116 patients). Unfortunately, we cannot recommend that polysomnography is withheld in patients with negative results from oximetry in the high probability group, as symptomatic patients with only minor oxygen desaturations would be missed. At the same time, polysomnography has to be performed in patients with positive results from oximetry in the low probability group, to detect nearly symptomless patients with sleep disordered breathing that could cause cardiovascular damage. These patients, however, may not accept effective treatment. ${ }^{20}$ 
Theoretically, patients with positive results from oximetry have to undergo polysomnography regardless of their probability group. Patients with full blown obstructive sleep apnoea, however, have characteristic cyclical oscillations of $\mathrm{SaO}_{2}$ and pulse rate throughout the night. About one third of such patients can be identified from visual scoring of oximetry alone. ${ }^{14}$ Our data support this as polysomnography would not have been absolutely necessary to confirm the diagnosis of obstructive sleep apnoea in the 17 subjects with an apnoea-hypopnoea index above 50 . Consequently, a total of 79 polysomnographic examinations would have been sufficient to manage our sample of 116 snorers, a reduction in the number of polysomnography studies of $32 \%$. In the light of recent results ${ }^{14}$ we might put forward the hypothesis that most of the remaining 79 polysomnography studies could be replaced by simplified sleep studies that include examination of breathing pattern and detection of leg movements as well; but the validity and cost effectiveness of this approach needs to be tested.

Unfortunately, we did not measure neck circumference, which has been shown to be a sensitive and highly specific predictor of obstructive sleep apnoea. ${ }^{2122}$ We suspect, however, that routine measurement of neck circumference will not reduce the number of sleep studies in snorers as physicians may be unwilling to refuse investigation on the basis of a normal neck circumference.

Given the discrepancy between the limited number of sleep laboratories in Europe today and the large number of patients referred for a sleep study, any attempt to reduce the number of expensive and time consuming polysomnography studies in snorers is useful. We conclude that snorers with negative results from oximetry classified as not having obstructive sleep apnoea by our prediction model do not need polysomnography. A further reduction of polysomnography studies in snorers seems feasible-by not doing polysomnography in patients of the high probability group with oximetric screening results unequivocally typical for obstructive sleep apnoea. In summary, such an approach should be able to reduce considerably the number of polysomnography studies in snor- ers without missing patients who would benefit from treatment.

1 Lugaresi E, Coccagna G, Farneti P, Mantovani M, Cirignotta F. Snoring. Electroencephalogr Clin Neurophysiol 1975;39:59-64.

2 Perez-Padilla JR, West P, Kryger M. Snoring in normal young adults: prevalence in sleep stages and associated changes in oxygen saturation, heart rate and breathing pattern. Sleep 1987;10:249-53.

3 Guilleminault C, Stoohs R, Duncan S. Snoring (I)Daytime sleepiness in regular heavy snorers. Chest 1991;99:40-8.

4 Hoffstein V, Mateika JH, Mateika MS. Snoring and sleep architecture. Am Rev Respir Dis 1991;143:92-6.

$5 \mathrm{He} J$, Kryger MH, Zorick FJ, Conway W, Roth T. Mortality and apnea index in obstructive sleep apnea. Experience in 385 male patients. Chest 1988;94:9-14.

6 Partinen M, Guilleminault C. Daytime sleepiness and vascular morbidity at seven-year follow-up in obstructive sleep apnea patients. Chest 1990;97:27-32.

7 Gonzalez-Rothi RJ, Foresman GE, Block AJ. Do patients with sleep apnea die in their sleep? Chest 1988; 94:531-8.

8 Partinen M, Jamieson A, Guilleminault C. Long-term outcome for obstructive sleep apnea syndrome patients. Chest 1988;94:1200-4.

9 Kapuniai L, Andrew D, Crowell D, Pearce J. Identifying sleep apnea from self-reports. Sleep 1988;11:430-6.

10 Crocker BD, Olson LG, Saunders NA, Hensley MJ, McKeon JL, Allen KM, et al. Estimation of the probability of disturbed breathing during sleep before a sleep study. Am Rev Respir Dis 1990;142:14-8.

11 Guilleminault C, Connoily S, Winkle R, Melvin K, Tilkian A. Cyclic variation of the heart rate in sleep apnea syndrome. Lancet 1984;i:126-31.

12 Farney RF, Walker LE, Jensen RL, Walker JM. Ear oximetry to detect apnea and differentiate rapid eye movement (REM) and non-REM (NREM) sleep. Chest 1986;89:533-9.

13 Cooper BG, Veale D, Griffiths CJ, Gibson GJ. Value of nocturnal oxygen saturation as a screening test for sleep apnoea. Thorax 1991;46:586-8.

14 Douglas NJ, Thomas S, Jan MA. Clinical value of polysomnography. Lancet 1992;339:347-50.

15 Gould GA, Whyte KF, Rhind GB, Airlie MA, Catterall JR, Shapiro CM, Douglas NJ. The sleep hypopnea syndrome. Am Rev Respir Dis 1988;137:895-8.

16 Wilhoit SC, Suratt PM, Evans RJ, Brown ED, Kaiser DL. Comparison of indices used to detect hypoventilation during sleep. Respiration 1985;47:237-42.

17 Rechtschaffen A, Kales A. A manual of standardized terminology techniques and scoring systems for sleep stages of human subjects. Washington DC: National Institute of Health, 1968. (Publication No 204.)

18 BMDP statistical software manual. Berkeley, California: University of California Press, 1985.

19 Rauscher H, Popp W, Zwick H. Computerized detection of respiratory events during sleep from rapid increases in oxyhemoglobin saturation. Lung 1991;169:135-42.

20 Rauscher H, Popp W, Wanke T, Zwick H. Acceptance of nasal CPAP therapy for sleep apnea. Chest 1991;100:1019-23.

21 Davies RJO, Ali N, Stradling JR. Neck circumference and other clinical features in the diagnosis of the obstructive sleep apnoea syndrome. Thorax 1992;47:101-5.

22 Davies RJO, Stradling JR. The relationship between neck circumference, radiographic pharyngeal anatomy, and the obstructive sleep apnoea syndrome. Eur Respir $\mathcal{f}$ 1990;3:509-14. 